\title{
Mechanical circulatory support in refractory cardiogenic shock due to influenza virus-related myocarditis
}

\author{
Jan-Thorben Sieweke (10) ${ }^{1,6}$, Muharrem Akin ${ }^{1,6}$, Sebastian Stetskamp \\ Christian Riehle ${ }^{1}$, Danny Jonigk ${ }^{2}$, Ulrike Flierl ${ }^{1}$, Tobias J. Pfeffer ${ }^{1}$, \\ Valentin Hirsch ${ }^{1}$, Jochen Dutzmann ${ }^{3}$, Marius M. Hoeper $\mathbb{1}^{4}$, Christian Kühn ${ }^{5}$, \\ Johann Bauersachs (10 ${ }^{1}$ and Andreas Schäfer ${ }^{1}$
}

Affiliations: ${ }^{1}$ Cardiac Arrest Center and Advanced Heart Failure Unit, Dept of Cardiology and Angiology, Hannover Medical School, Hannover, Germany. ${ }^{2}$ Dept of Pathology, Hannover Medical School, Hannover, Germany. ${ }^{3}$ Mid-German Heart Center, Dept of Cardiology, Angiology, and Intensive Care Medicine, University Hospital, Martin Luther University Halle-Wittenberg, Halle (Saale), Germany. ${ }^{4}$ Dept of Respiratory Medicine and the German Center for Lung Research (DZL), Hannover Medical School, Hannover, Germany. ${ }^{5}$ Dept of Cardiothoracic, Transplant and Vascular Surgery, Hannover Medical School, Hannover, Germany. ${ }^{6}$ These authors contributed equally to the manuscript.

Correspondence: Jan-Thorben Sieweke, Dept of Cardiology and Angiology, Hannover Medical School, Carl-Neuberg-Straße 1, 30625 Hannover, Germany. E-mail: sieweke.jan-thorbenlamh-hannover.de

@ERSpublications

Combined mechanical circulatory support in refractory cardiogenic shock (ECMELLA) might not salvage patients with influenza-associated myocarditis and severe end-organ damage, in contrast to the favourable effects in primary cardiac causes https://bit.ly/3dmonC4

Cite this article as: Sieweke J-T, Akin M, Stetskamp S, et al. Mechanical circulatory support in refractory cardiogenic shock due to influenza virus-related myocarditis. Eur Respir J 2020; 56: 2000925 [https://doi. org/10.1183/13993003.00925-2020].

\section{ABSTRACT}

Background: There is scarce evidence for mechanical circulatory support (MCS) in patients with influenza-related myocarditis complicated by refractory cardiogenic shock (rCS). We sought to investigate the impact of MCS using combined veno-arterial extracorporeal membrane oxygenation (VA-ECMO) and micro-axial flow pumps (the ECMELLA concept) in influenza-related myocarditis complicated by rCS.

Methods: This is a prospective, observational analysis from the single centre HAnnover Cardiac Unloading REgistry (HACURE) from two recent epidemic influenza seasons. We analysed patients with verified influenza-associated myocarditis complicated by rCS who were admitted to our intensive care unit (ICU) on MCS. Subsequently, we performed a propensity score (PS) matched analysis to patients with acute myocardial infarction (AMI) complicated by rCS and non-ischaemic cardiomyopathy (DCM) related rCS.

Results: We describe a series of seven patients with rCS-complicated influenza-related myocarditis (mean age 56 \pm 10 years, $58 \%$ male, influenza A $(n=2)$ /influenza $B(n=5))$. No patient had been vaccinated prior to the influenza season. MCS was provided using combined VA-ECMO and Impella micro-axial flow pump. In two patients with out-of-hospital cardiac arrest, VA-ECMO had been implanted for extracorporeal cardiopulmonary resuscitation. All patients died within 18 days of hospital admission. By PS-based comparison to patients with AMI- or DCMrelated rCS and combined MCS, 30-day mortality was significantly higher in influenza-related rCS.

Conclusion: Despite initial stabilisation with combined MCS in patients with rCS-complicated influenza-related myocarditis, the detrimental course of shock could not be stopped and all patients died. Influenza virus infection potentially critically affects other organs besides the heart, leading to irreversible end-organ damage that MCS cannot compensate for and, therefore, results in a devastating outcome.

This article has an editorial commentary: https://doi.org/10.1183/13993003.02129-2020

This article has supplementary material available from erj.ersjournals.com

Received: 3 April 2020 | Accepted after revision: 1 May 2020

Copyright OERS 2020. This version is distributed under the terms of the Creative Commons Attribution NonCommercial Licence 4.0. 


\section{Introduction}

Influenza virus commonly causes seasonal respiratory infections and periodically leads to epidemics and pandemics. Respiratory failure and pneumonia are common respiratory complications and are associated with impaired survival [1]. The myocardium is affected in about $10 \%$ of cases, usually starting between Day 4 and Day 7 after symptom onset $[1,2]$. Myocardial manifestations are underdiagnosed due to variability, non-specificity and onset of symptoms [3]. Definite acute myocarditis was diagnosed in one third of 33 cases with unexpected death without prior suspicion of myocardial involvement during the Asian influenza pandemic of 1957 [4]. Influenza-related myocarditis presents in a variable manner, from subclinical to fulminant myocarditis with or without overt cardiogenic shock (CS), which can ultimately result in cardiac failure leading to death [1]. CS-complicated influenza-related myocarditis is scarce and, in their systematic review of 184 cases of myocarditis-complicated influenza infection, HékIMIAN et al. [5] reported 48 CS cases treated with mechanical circulatory support (MCS).

Catecholamines are recommended by guidelines to stabilise blood pressure in patients with CS, but contribute to secondary multi-organ failure due to systemic vasoconstriction [6, 7]. In refractory cardiogenic shock (rCS), percutaneous MCS is considered as an optional treatment for patients unresponsive to fluid load and vasopressors [7]. In this analysis we sought to investigate the efficacy of MCS with combined veno-arterial extracorporeal membrane oxygenation (VA-ECMO) and Impella micro-axial flow pump (the ECMELLA concept) in rCS-complicated influenza-related myocarditis.

\section{Methods}

\section{Study design and participants}

Our analysis was performed in accordance with the Declaration of Helsinki and has been approved by the local ethics committee (\#3566-2017). In the analysis, all patients with rCS-complicated influenza who were admitted to the Department of Cardiology at Hannover Medical School during two recent influenza epidemic seasons (2013 and 2018) and who were treated with MCS using VA-ECMO and Impella micro-axial flow pump, were analysed. Of 400 consecutive patients treated with micro-axial flow pumps included in a local database (the HAnnover Cardiac Unloading REgistry (HACURE) [8]), those who had received MCS with combined VA-ECMO and Impella micro-axial flow pump for rCS due to acute myocardial infarction (AMI) or non-ischaemic cardiomyopathy (DCM) from January 2013 to June 2018, labelled as the AMI-rCS group and the DCM-rCS group, respectively, were considered for propensity score (PS) matching as described in figure 1. Shock severity score at admission, the Survival After Veno-arterial ECMO (SAVE) score [9], demographic data, laboratory data and complications during in-hospital stay were recorded.

\section{Patient treatment and definitions}

Influenza infection was confirmed by PCR analysis of pharyngeal swab tests. rCS was defined as persistence or deterioration of hypotension and/or end-organ hypoperfusion, i.e. elevated lactate levels $\left(\geqslant 2.5 \mathrm{mmol} \cdot \mathrm{L}^{-1}\right)$ plus one or more clinical sign of hypoperfusion (e.g. clammy skin, tachycardia, altered mental status, oliguria $\left(<30 \mathrm{~mL} \cdot \mathrm{h}^{-1}\right)$, or pulmonary oedema), despite catecholamine administration (weight-adjusted maximal dosing of two or more catecholamines) and appropriate standard shock therapy according to groups D and E of the Society for Cardiovascular Angiography and Interventions (SCAI) clinical expert consensus statement [10,11]. Patients were treated according to current guidelines [7] and a local treatment algorithm (HaCRA) for CS and cardiac arrest [12]. Detailed patient treatment information is given in figure 2 and in the supplementary material.

\section{Statistical analysis}

Categorical parameters are given as $\mathrm{n}(\%)$, normally distributed metric variables are presented as mean \pm standard deviation (SD) and non-normally distributed metric variables as median (interquartile range (IQR)). Comparison between time points was performed with ANOVA and the Mann-Whitney U-test as a nonparametric test, followed by correction for multiple comparisons by the Bonferroni test or Dunn's test. Statistical analyses for comparison between PS-matched groups of metric parameters were performed using unpaired t-tests as parametric tests and Mann-Whitney tests as nonparametric tests. The Chi-squared test was applied to compare nominally scaled parameters. Thirty-day survival was calculated using Kaplan-Meier curves and performing log-rank comparisons between the groups. Cox regressions analysis was performed to calculate hazard ratios with 95\% confidence intervals (CIs). Reported p-values are two-sided, with $\mathrm{p}<0.05$ considered statistically significant. Data were analysed using GraphPad Prism version 7.04 (GraphPad Software, San Diego, CA, USA), R version 3.3.3 (The R Foundation for Statistical Computing, www.r-project.org) and SPSS Statistics version 25 (IBM Inc, Armonk, NY, USA). Detailed PS matching is presented in the supplementary material and supplementary figure S1. 


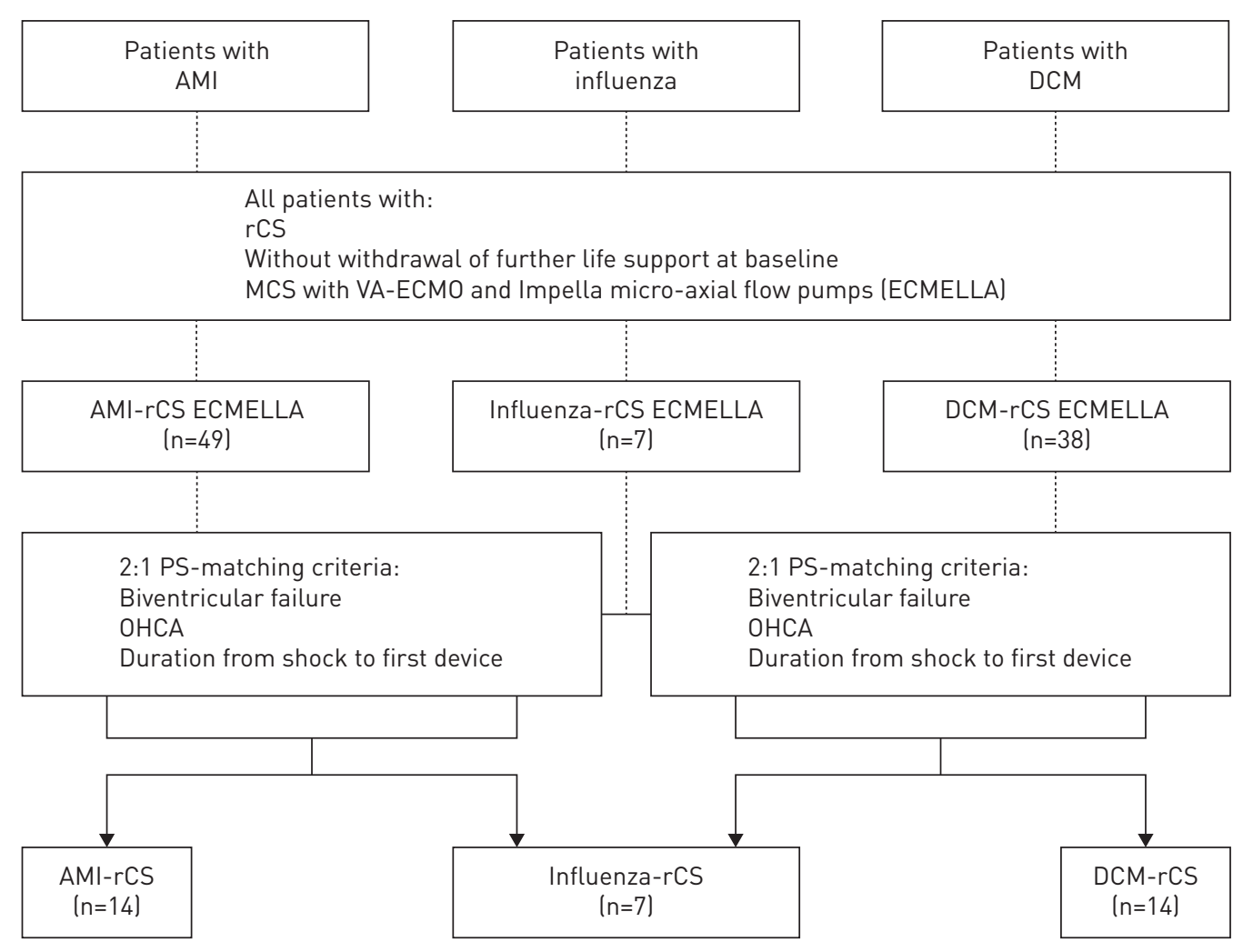

FIGURE 1 Flow diagram of study enrolment. AMI: acute myocardial infarction; DCM: non-ischaemic cardiomyopathy; rCS: refractory cardiogenic shock; AMI-rCS: AMI-related rCS; influenza-rCS: influenza-related rCS; DCM-rCS: DCM-related rCS; MCS: mechanical circulatory support; VA-ECMO: veno-arterial extracorporeal membrane oxygenation; ECMELLA: MCS with combined VA-ECMO and Impella micro-axial flow pump; PS: propensity score; OHCA: out-of-hospital cardiac arrest.

\section{Results}

Patient characteristics

A total of seven Caucasian patients without prior influenza vaccination and with rCS-complicated influenza infection, who were treated with MCS, were analysed (mean age: 56 \pm 10 years). PCR analysis confirmed influenza B in five patients and influenza $\mathrm{A}$ in two patients (numbers four and seven). In four patients (numbers one to four), left-ventricular endomyocardial biopsy had been performed. Histological examination revealed interstitial lymphocytic infiltration and focal myocyte necrosis (figure 3). Myocarditis was clinically diagnosed based on symptoms, cardiac enzyme elevation and echocardiographic findings [13] in the other three patients. A median SAVE score of -11 (IQR -12 to -8 ) indicated an estimated mortality of $82 \%$ even when supported on VA-ECMO. Between January 01, 2013 and June 30, 2018, a total of 87 patients with AMI-related rCS $(n=49)$ or DCM-related $\operatorname{rCS}(n=38)$ had been treated with ECMELLA at our institution. After 1:2 PS matching, 14 patients from the AMI-related rCS group (the "AMI-rCS group") and 14 patients from the DCM-related rCS group (the "DCM-rCS group") were included for further comparison as described in figure 1. Baseline characteristics between the groups did not differ in a statistically significant way. Patient characteristics are shown in table 1 and in supplementary table S1.

\section{Intensive care and MCS characteristics}

During a median intensive care unit (ICU) stay of 3 days (IQR 1-16 days) all patients were mechanically ventilated. Transfer from a referral hospital had been performed on VA-ECMO in two patients (numbers three and four). Four patients suffered from cardiac arrest prior to hospital admission. All resuscitated patients had witnessed arrest and bystander resuscitation. VA-ECMO was implanted for extracorporeal CPR (eCPR) in two patients (numbers one and six). Respiratory failure due to acute respiratory distress syndrome (ARDS) occurred in two patients (numbers three and four) resulting in escalation to veno-arterial venous extracorporeal membrane oxygenation (VAV-ECMO) [14]. Impella micro-axial flow pumps were inserted secondary to VA-ECMO based on left-ventricular distension and pulmonary congestion in two patients (numbers one and six). Admission lactate level, frequency of cardiac arrest and 


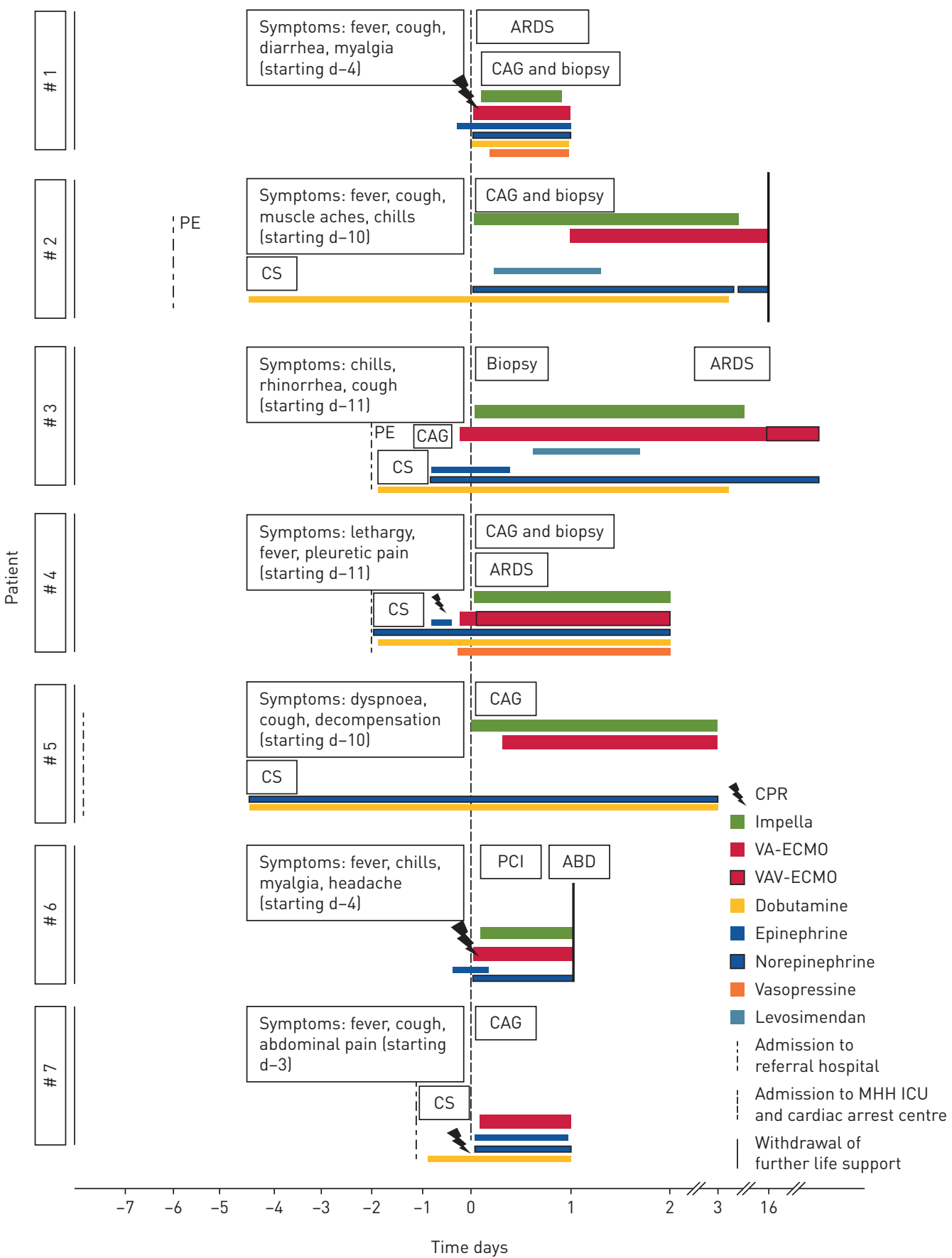

FIGURE 2 Time course of treatment in patients with refractory cardiogenic shock (rCS)-complicated myocarditis induced by influenza virus infection. ARDS: acute respiratory distress syndrome; CAG: coronary angiography; CS: cardiogenic shock; PE: pericardial effusion; PCI: percutaneous coronary intervention; ABD: anoxic brain damage; CPR: cardio-pulmonary resuscitation; VA-ECMO: veno-arterial extracorporeal membrane oxygenation; VAV-ECMO: veno-arterial venous extracorporeal membrane oxygenation; $\mathrm{MHH}$ : Hannover Medical School; ICU: intensive care unit.

duration from shock onset to either first device, VA-ECMO, or Impella micro-axial flow pump use did not differ significantly between the matched groups. Clinical course based on ICU and MCS characteristics is summarised in table 2 and supplementary table S2. MCS provided haemodynamic stabilisation (figures 4a and b), resulting in decreased infusion of inotropes and vasopressors (figure 4c) in patients with influenza-related myocarditis-associated rCS. Lactate levels declined rapidly during MCS and increased in two patients after left-ventricular recovery, probably based on subsequent sepsis following pneumonia and ARDS (figure $4 \mathrm{~d}$ ). 

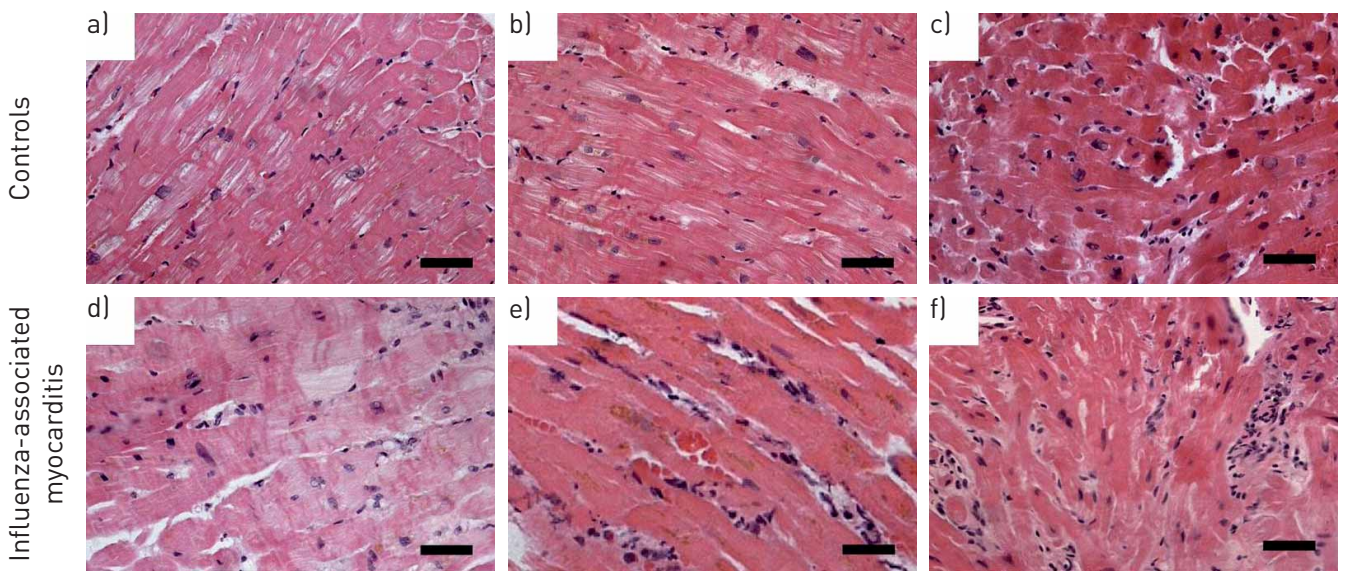

FIGURE 3 Endomyocardial biopsies of patients supported by percutaneous mechanical circulatory support (MCS) with refractory cardiogenic shock (rCS)-complicated non-ischaemic cardiomyopathy (DCM) and influenza-related myocarditis. (a-c) Controls: cardiac left-ventricular biopsies of patients with rCS-complicated DCM with percutaneous MCS. In these endomyocardial biopsies cardiomyocytes show signs of irregular hypertrophy with varying hyperchromasia of the corresponding nuclei. Also present are unevenly dispersed, mildly eosinophilic contraction bands, as well as a mild intracellular and extracellular oedema. While there is some sparse interstitial inflammatory infiltration, the criteria of an active myocarditis laccording to the Dallas classification) are not met. By definition, the histological changes in dilated cardiomyopathy are nonspecific, rendering the histopathological diagnosis one of exclusion. Signs of specific disorders, such as granulomatous inflammation, myocardial inclusions or siderosis are absent. (d-f) Influenza-associated myocarditis: endomyocardial biopsies of patients with rCS-complicated influenza-related active myocarditis. There is a pronounced if unevenly distributed interstitial inflammatory infiltrate, for the most part made up of activated T-lymphocytes. All biopsies show evidence of myocyte damage, which ranges from prominent contraction bands to hyper-eosinophilic (early) stages of necrosis. Adjacent capillaries are dilated, packed with erythrocytes and their endothelial nuclei are activated. Cardiomyocytes as well as the cardiac interstitium show accompanying oedematous changes. As in (a-c), signs of specific disorders such as granulomatous inflammation, myocardial inclusions or siderosis are absent. Scale bars $=50 \mu \mathrm{m}$.

\section{Safety outcome}

In one patient (number seven), the Impella micro-axial flow pump could not be repositioned in the left ventricle after dislocation to the ascending aorta. In two patients (numbers two and three), the Impella micro-axial flow pumps were explanted after primary left-ventricular recovery (following active

TABLE 1 Patient characteristics in the study

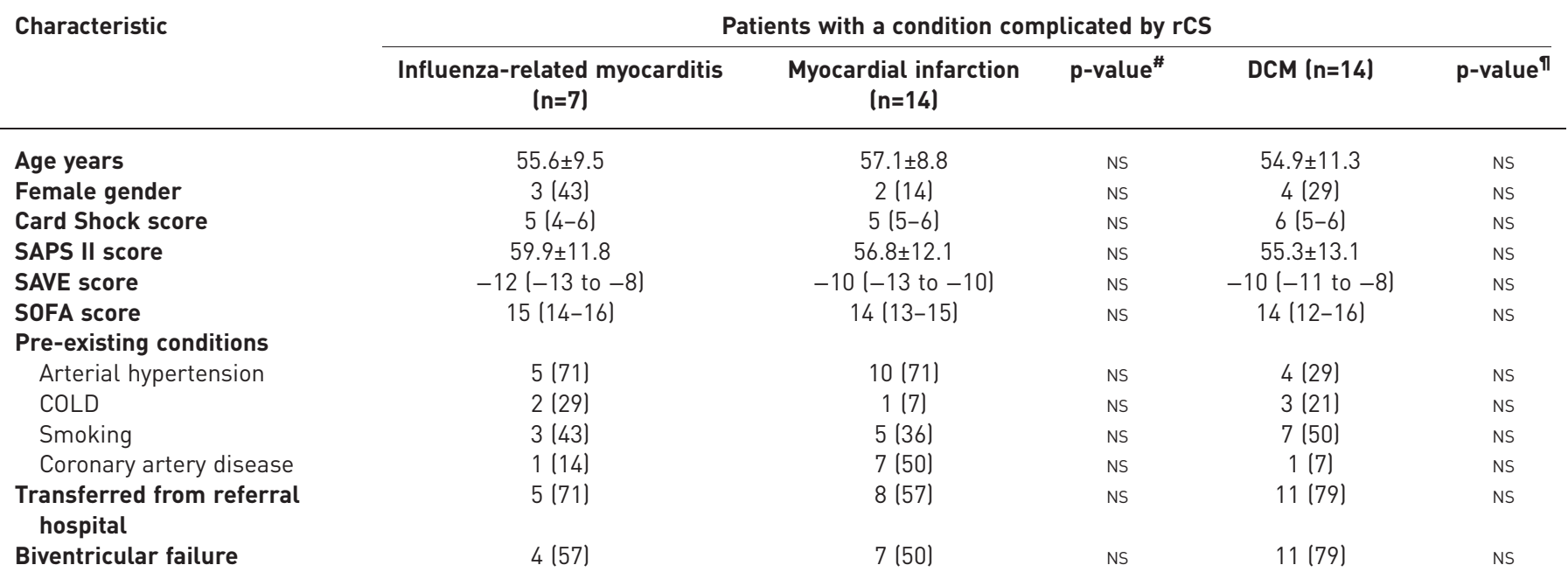

Data are presented as $n(\%)$, mean \pm SD, or median (IQR). DCM: non-ischaemic cardiomyopathy; NS: not statistically significant; SAPS: Simplified Acute Physiology Score; SAVE: Survival After Veno-arterial ECMO; SOFA: Sequential Organ Failure Assessment; COLD: chronic obstructive lung disease; SD: standard deviation; IQR: interquartile range. AMI: acute myocardial infarction; rCS: refractory cardiogenic shock; AMI-rCS: AMI-related rCS; DCM-rCS: DCM-related rCS; influenza-rCS: influenza-related rCS. \#: influenza-rCS versus AMI-rCS; "ः influenza-rCS versus DCM-rCS. 
TABLE 2 Intensive care and mechanical circulatory support (MCS)

\begin{tabular}{|c|c|c|c|c|c|}
\hline & \multicolumn{5}{|c|}{ Patients with a condition complicated by rCS } \\
\hline & $\begin{array}{l}\text { Influenza-related myocarditis } \\
\qquad(n=7)\end{array}$ & $\begin{array}{l}\text { Myocardial infarction } \\
\qquad(n=14)\end{array}$ & p-value $\#$ & $\begin{array}{l}\text { DCM } \\
(n=14)\end{array}$ & p-value ${ }^{\pi}$ \\
\hline OHCA & $2(29)$ & $7(50)$ & NS & $4(29)$ & NS \\
\hline Initial rhythm (VT/VF) & $2(100)$ & $7(100)$ & NS & $4(100)$ & NS \\
\hline Witnessed arrest & $4(57)$ & $9(64)$ & NS & $4(29)$ & NS \\
\hline eCPR & $2(29)$ & $2(14)$ & NS & $1(7)$ & NS \\
\hline \multicolumn{6}{|l|}{ MCS } \\
\hline Escalation to VAV-ECMO & $2(29)$ & $2(14)$ & NS & 0 & 0.035 \\
\hline $\begin{array}{l}\text { Biventricular support with Impella } \\
\text { micro-axial flow pump and VA-ECMO }\end{array}$ & $7(100)$ & $14(100)$ & NS & $14(100)$ & NS \\
\hline Duration from shock to first device min & $20(2-32)$ & $6(4-22)$ & NS & $17(5-28)$ & NS \\
\hline Secondary pneumonia & $2(29)$ & $0(0)$ & 0.035 & $0(0)$ & 0.035 \\
\hline Secondary ARDS & $3(43)$ & $2(14)$ & NS & $0(0)$ & 0.008 \\
\hline$A K I$ at admission & $6(86)$ & $12(86)$ & NS & $12(86)$ & NS \\
\hline Renal replacement therapy & $6(86)$ & $9(64)$ & NS & $7(50)$ & NS \\
\hline 30-day mortality & $7(100)$ & $6(43)$ & 0.011 & $7(50)$ & 0.035 \\
\hline
\end{tabular}

Data are presented as $\mathrm{n}(\%)$ or median (IQR). p-Values in bold indicate statistical significance. DCM: non-ischaemic cardiomyopathy; NS: not statistically significant; OHCA: out-of-hospital cardiac arrest; VT: ventricular tachycardia; VF: ventricular fibrillation; CPR: cardio-pulmonary resuscitation; ROSC: return of spontaneous circulation; eCPR: extracorporeal CPR; VAV-ECMO: veno-arterial venous extracorporeal membrane oxygenation; ECMO: extracorporeal membrane oxygenation; LVAD: left-ventricular assist device (durable); ARDS: acute respiratory distress syndrome; AKI: acute kidney injury. AMI: acute myocardial infarction; rCS: refractory cardiogenic shock; influenza-rCS: influenza-related rCS; AMI-rCS: AMI-related rCS; DCM-rCS: DCM-related rCS. " : influenza-rCS versus AMI-rCS; ๆ : influenza-rCS versus DCM-rCS.

left-ventricular unloading of $369 \mathrm{~h}$ (number two) and $312 \mathrm{~h}$ (number three)). Pulmonary influenza infection was complicated by ARDS in three patients (numbers one, three and four) and by secondary bacterial pneumonia in two patients (numbers two and five), with consecutive septic shock. Liver failure at admission was present in five patients (71\%; number one and numbers four to seven). No patient survived for 30 days after hospital admission (figure 5). Withdrawal of further life support was decided upon by a consensus view due to multi-organ failure and protracted rCS (patient number two) and distinct anoxic brain damage $(\mathrm{ABD})$ following resuscitation (patient number six) (figure 2). The remaining five patients (numbers one, three to five and seven) died while on maximal escalated intensive care. During MCS, no apparent thromboembolic events occurred.

Patients with influenza-related myocarditis-associated rCS suffered more frequently from secondary pneumonia and subsequent respiratory failure necessitating escalation to VAV-ECMO compared to the DCM-rCS group. Safety outcomes are summarised in table 2, supplementary figure S2 and supplementary table S3.

\section{0-Day mortality in propensity score matched groups}

In PS-matched groups, $57 \%$ of patients in the AMI-rCS group $(n=8)$ and $50 \%$ in the DCM-rCS group $(n=7)$ survived until Day-30 after ICU admission, while their predicted mortality on VA-ECMO, based on the SAVE score, had been $82 \%$ for both groups. All patients with influenza-related myocarditis-associated rCS died within 18 days. Thus, 30-day survival was significantly different between the influenza-rCS group $(\mathrm{n}=7)$ and both the matched AMI-rCS group (hazard ratio 3.56,95\% CI 1.07-12.83; p=0.006; $\mathrm{n}=14$ ) and the DCM-rCS group (hazard ratio 3.39, 95\% CI 1.30-13.86; $\mathrm{p}=0.003$; $\mathrm{n}=14$ ). Kaplan-Meier curves are provided in figure 5 .

\section{Review of the literature}

A detailed literature review is provided in the supplementary material. We identified 10 cases of which $70 \%(\mathrm{n}=7)$ were female. Mean age was $39.5 \pm 13.2$ years and influenza type was determined in each case 

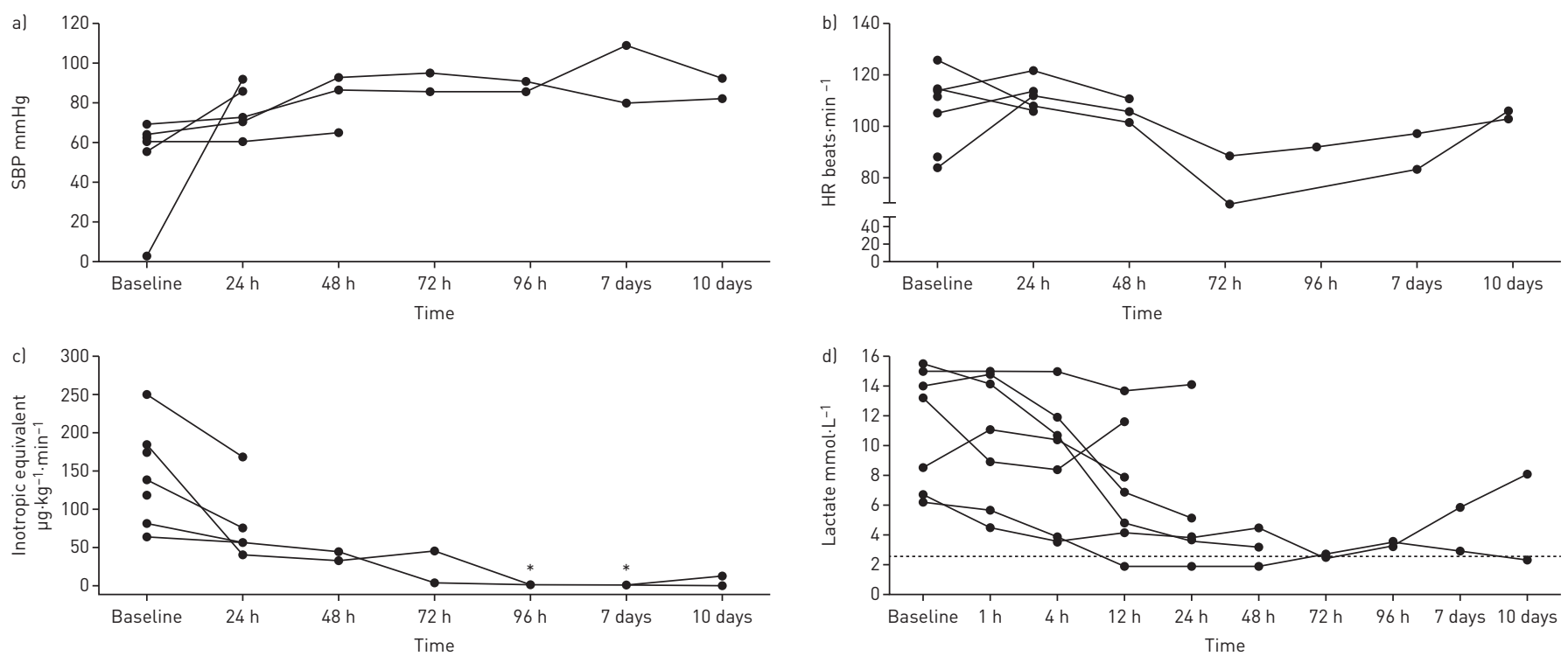

FIGURE 4 Haemodynamic effects of mechanical circulatory support (MCS) in patients with refractory cardiogenic shock (rCS)-complicated myocarditis induced by influenza virus infection (where a) systolic blood pressure (SBP); b) heart rate (HR); c) inotropic equivalent level; d) lactate level). Despite stabilisation of haemodynamic parameters (with consequent decrease of the inotropic equivalent level) and counteracting of rCS status (with consequent decrease of the lactate level), based on percutaneous MCS, patients died within 18 days of admission to the intensive care unit (ICU) and cardiac arrest centre of Hannover Medical School (MHH). Catecholamine dose was evaluated by the inotrope equivalent method

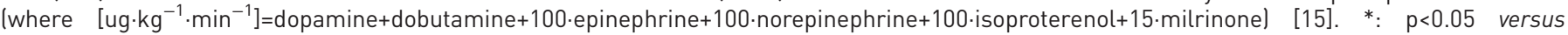
baseline.

(influenza A $(n=2)$ and influenza B $(n=8))$. These patients were supported with VA-ECMO $(n=8)$ or Impella micro-axial flow pump $(\mathrm{n}=2)$ (ECMELLA $(\mathrm{n}=0)$ ), with an in-hospital survival rate of $80 \%$ as provided in supplementary table S4. Two patients (20\%) fulfilled the applied definitions of rCS [10, 11]. A prior influenza vaccination was not reported in any case. Three patients were in need for renal replacement therapy and two patients (20\%) suffered cardiac arrest. No patients suffered from secondary pneumonia or ARDS and escalation of ECMO cannulation strategy was not performed.

\section{Discussion}

Our present analysis includes seven patients with rCS-complicated influenza-related myocarditis treated with combined Impella micro-axial flow pump and VA-ECMO circulatory support (the ECMELLA concept). Based on the extent of shock, as determined by the SAVE score (median -11, IQR -12 to -8 ), these patients were at extreme risk of death. The ECMELLA strategy $[16,17]$ obviously improved

a)

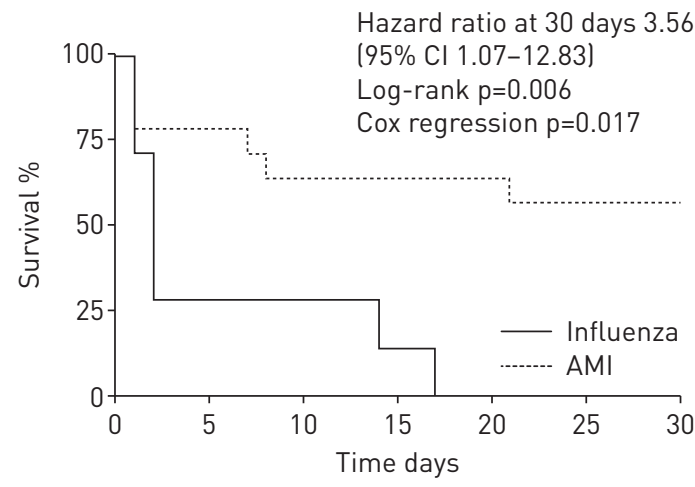

b)

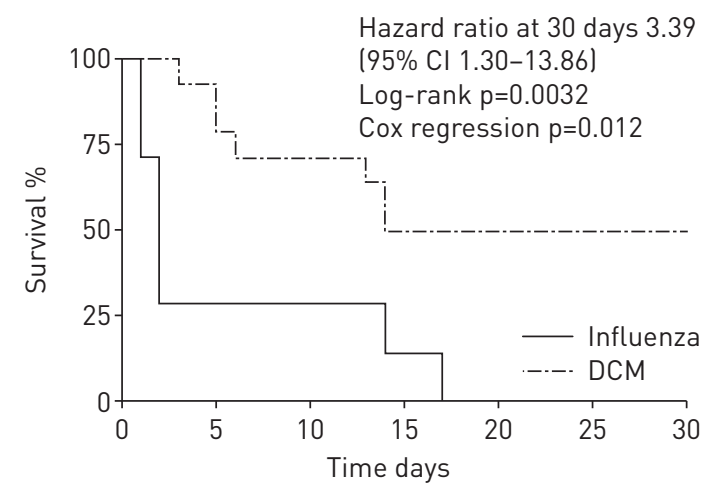

Number at risk: Influenza-rCS AMI-rCS

$\begin{array}{ccccccc}7 & 2 & 2 & 1 & 0 & 0 & 0 \\ 14 & 11 & 9 & 9 & 9 & 8 & 8\end{array}$

Number at risk:

Influenza-rCS

DCM-rCS

$\begin{array}{ccccccc}7 & 2 & 2 & 1 & 0 & 0 & 0 \\ 14 & 11 & 10 & 7 & 7 & 7 & 7\end{array}$

FIGURE 5 30-Day survival of propensity score (PS) matched cohorts. a) Matching of influenza-rCS and AMI-rCS. b) Matching of influenza-rCS and DCM-rCS. Cl: confidence interval; AMI: acute myocardial infarction; DCM: non-ischaemic cardiomyopathy; rCS: refractory cardiogenic shock; AMI-rCS: AMI-related rCS; DCM-rCS: DCM-related rCS; influenza-rCS: influenza-related rCS. 
haemodynamic compromise in these patients, reduced the need for inotropes and led to Impella micro-axial flow pump weaning in two patients after left-ventricular recovery. However, despite initial haemo-metabolic improvement by ECMELLA, none of our severely compromised patients with influenza-related, myocarditis-associated rCS survived. When applying PS matching to patient cohorts with AMI-related rCS or DCM-related rCS, to account for haemodynamic compromise, patients with influenza-related, myocarditis-associated rCS showed a significantly higher 30-day mortality rate. Patients with rCS-complicated, influenza-related myocarditis suffered from irreversible end-organ failure complicated by cardiac arrest $(n=4,57 \%)$ and/or liver failure $(n=5,71.4 \%)$, which was not necessarily a consequence of rCS but rather a direct extra-cardiac organ injury caused by the influenza infection itself. Therefore, combined MCS with ECMELLA may have had a minor impact on prognosis in these patients, in comparison to patients with either AMI or DCM resulting in rCS. In these entities, symptoms of end-organ failure are most probably a consequence of haemodynamic deterioration. In contrast, influenza infection causes multiple extra-cardiac injuries that are independent of haemodynamic deterioration and haemodynamic stabilisation by MCS is unable to improve end-organ injury. MCS strategies have yielded a promising therapeutic concept with which to interrupt the fatal consequences of $\operatorname{rCS}[18,19]$. Due to the lack of evidence for many MCS devices in general and their application in rarer diseases such as rCS in myocarditis in particular, decision making with respect to MCS is an individual process based on local experience.

In patients with rCS, MCS by combined treatment (ECMELLA) has been associated with improved outcomes compared to singular VA-ECMO treatment [20]. Notably, that retrospective analysis also included patients with myocarditis-associated rCS, but probably only involved infections restricted to the heart itself. To the best of our knowledge, the MCS approach of either VA-ECMO or Impella micro-axial flow pump in CS-complicated, influenza-related myocarditis is limited to sporadic case reports [5, 21, 22].

In our analysis, five patients $(71 \%)$ were transferred from referral hospitals to our department after some delay (median duration from shock to first device $26 \mathrm{~h}, \mathrm{IQR} 18-40 \mathrm{~h}$ ). Early diagnosis of myocarditis in the case of a concomitant influenza infection is challenging based on the length of the disease course prior to hospital admission, variability in clinical presentation, comorbidities and challenges in influenza virus detection [23, 24]. This might delay the transfer of severely compromised patients to tertiary hospitals. Furthermore, invasive haemodynamic monitoring is not commonly performed in all patients with borderline or compensated CS. An obvious contributor to counteracting the cardio-metabolic deterioration of CS is door to support time $[8,25]$. Therefore, we considered duration from shock to first device in PS-matching, in addition to biventricular failure at admission and out-of hospital cardiac arrest (OHCA). Both matched cohorts showed a significantly higher survival rate compared to the influenza cohort within 30 days of ICU admission and as predicted by the SAVE score.

The ECMELLA concept, as well as the standardised care on our ICU, was thus clearly able to benefit extremely compromised rCS patients. However, patients suffering from influenza-related, myocarditis-associated rCS did not profit from haemodynamic stabilisation. Cardiac end-organ damage owing to influenza-related, rCS-complicated myocarditis is presented in left-ventricular endomyocardial biopsies (figure 3). Additionally, the severity of myocardial damage in influenza-related myocarditis is enhanced owing to cytokines and an overwhelming inflammatory response [24, 26-28]. We concluded that in these patients the probability of myocardial recovery was less than expected in comparison to the PS-matched DCM cohort.

On first sight, our results seem to be in contrast to previous case reports, which indicate a low mortality rate in patients with influenza-related, CS-complicated myocarditis supported by VA-ECMO or Impella micro-axial flow pump (supplementary table S4). Overall, the variance of in-hospital survival of patients with influenza-related myocarditis between our cohort and the historic patient examples may be explained by their younger age (55.6 \pm 9.5 years versus $39.5 \pm 13.2$ years), absence of additional influenza-related complications, the severity of CS and subsequent differences in end-organ damage.

Similarly, in the current pandemia of another challenging RNA-virus (severe acute respiratory syndrome coronavirus 2 (SARS-CoV-2)), older age and comorbidities, as well as virus-related complications and end-organ damage, are associated with impaired outcome [29]. The infection presents with influenza-like symptoms (e.g. fever, cough, headache, fatigue, myalgia, and shortness of breath) and can ultimately result in multi-organ dysfunction involving ARDS, acute liver failure, acute kidney injury (AKI) and acute heart failure [29-31]. Two recently published articles describe coronavirus disease 2019 (COVID-19) associated myocardial injury detected by elevated high-sensitivity troponin I and troponin T levels [32, 33]. Notably, in both of these analyses, myocardial injury was associated with a higher degree of in-hospital mortality. In a similar fashion to influenza virus infection, the cases reported by INCIARDI et al. [34] describe myocardial injury that might be represented by acute myocarditis. However, due to scarce reports of cases 
of MCS in rCS related to confirmed COVID-19 diagnoses, the effect of ECMELLA on mortality in these patients cannot be extrapolated from our analysis and, therefore, remains uncertain $[29,35]$. Nevertheless, the experience of futile causes gained in influenza-related, myocarditis-associated rCS patients, due to the extra-cardiac end-organ damage caused by the virus, might well be applicable in the case of COVID-19.

In summary, all cases of rCS related to influenza-infection were observed in non-vaccinated patients over two epidemic seasons. Therefore, physicians should emphasise the importance of influenza vaccination in both healthy people and in patients with pre-existing cardiac disease [36]. Furthermore, AMI- and DCM-related rCS affect primarily cardiomyocytes selectively, while influenza infection secondarily affects the heart by a systemic inflammatory response while it also hits other vital organs. Thus, influenza causes severe end-organ damage that might not be salvageable by MCS, in contrast to the beneficial effects observed in the primary cardiac causes of rCS. As such, MCS should be considered carefully in patients with influenza-related myocarditis-associated rCS, in particular in relation to the fatal outcome. However, without indicators for the irreversible stage of end-organ failure or the diagnosis of influenza infection in these patients, MCS strategies seem to be the only option to treat acute haemodynamic deterioration and prevent early death. Further analyses of ECMELLA support in patients with influenza-associated, myocarditis-related rCS are warranted to verify our hypothesis.

\section{Limitations}

Based on the retrospective and observational design, no randomised control group is available. The observational registry reports only a small series of patients with rCS-complicated influenza virus infection. Registries provide relevant information for therapeutic approaches in rare diseases and, while the results may be hypothesis generating, patients with influenza-associated, myocarditis-related rCS are scarce and represent a subset of severely compromised patients. Therefore, we cannot extrapolate in this analysis on whether an earlier implantation of MCS would have resulted in a beneficial effect and to what extent respiratory failure, ARDS and secondary pneumonia contributed to mortality. Furthermore, the results of PS-matching with small patient numbers can only be carefully extrapolated based on possible biases regarding potentially unknown covariates [37].

\section{Conclusions}

We show that rCS in influenza-related myocarditis seems to have a fatal course despite implantation of combined MCS. As such our data do not support the concept of standardised aggressive use of MCS in these patients. Nevertheless, the alternative without MCS being an immediate fatal outcome will often trigger implantation; however, this might not influence prognosis in a relevant fashion.

Acknowledgements: We thank the nursing staff of the ICU and the cardiac arrest centre (Dept of Cardiology and Angiology, Hannover Medical School) for supporting this study.

Author contributions: J-T. Sieweke and M. Akin contributed equally to the manuscript. J-T. Sieweke, A. Schäfer and J. Bauersachs designed the registry. J-T. Sieweke, M. Akin, S. Stetskamp, C. Riehle, U. Flierl, T.J. Pfeffer, V. Hirsch, J. Dutzmann, M.M. Hoeper and C. Kühn recruited patients and collected data. J-T. Sieweke, M. Akin and S. Stetskamp analysed and interpreted data. J-T. Sieweke, M. Akin, C. Riehle, J. Bauersachs and A. Schäfer wrote the manuscript. J-T. Sieweke, M. Akin, U. Flierl, J. Dutzmann and A. Schäfer performed the literature review. D. Jonigk rated and analysed the endomyocardial biopsies of patients supported by MCS with influenza-rCS and DCM-rCS. All authors revised the manuscript critically. J. Bauersachs and A. Schäfer accurately approved the manuscript.

Conflict of interest: J-T. Sieweke reports travel support from Abiomed, outside the submitted work. M. Akin has nothing to disclose. S. Stetskamp has nothing to disclose. C. Riehle reports travel support from Abiomed, outside the submitted work. D. Jonigk has nothing to disclose. U. Flierl has nothing to disclose. T.J. Pfeffer has nothing to disclose. V. Hirsch has nothing to disclose. J. Dutzmann has nothing to disclose. M.M. Hoeper reports personal fees for lectures and consultancy work from Actelion, Bayer, MSD and Pfizer, outside the submitted work. C. Kühn has nothing to disclose. J. Bauersachs reports personal fees from Novartis, BMS, Pfizer, Bayer, Servier, MSD, Boehringer Ingelheim, AstraZeneca, Abbott, Medtronic and Daiichi Sankyo, grants and personal fees from Abiomed, Zoll, Vifor and CvRX, outside the submitted work. A. Schäfer reports grants and personal fees from Abiomed, during the conduct of the study.

Support statement: This study was supported by the Klinische Forschergruppe (KFO, award 311-TP1) and the Deutsche Forschungsgemeinschaft (DFG, award BA 1742/9-1). Funding information for this article has been deposited with the Crossref Funder Registry.

\section{References}

1 Estabragh ZR, Mamas MA. The cardiovascular manifestations of influenza: a systematic review. Int J Cardiol 2013; 167: $2397-2403$.

2 Onitsuka $\mathrm{H}$, Imamura $\mathrm{T}$, Miyamoto $\mathrm{N}$, et al. Clinical manifestations of influenza a myocarditis during the influenza epidemic of winter 1998-1999. J Cardiol 2001; 37: 315-323.

3 Rezkalla SH, Kloner RA. Influenza-related viral myocarditis. WMJ 2010; 109: 209-213. 
4 Oseasohn R, Adelson L, Kaji M. Clinicopathologic study of thirty three fatal cases of Asian influenza. $N$ Engl J Med 1959; 260: 509-518.

5 Hekimian G, Jovanovic T, Brechot N, et al. When the heart gets the flu: fulminant influenza B myocarditis: a case-series report and review of the literature. J Crit Care 2018; 47: 61-64.

6 den Uil CA, Lagrand WK, van der Ent M, et al. Impaired microcirculation predicts poor outcome of patients with acute myocardial infarction complicated by cardiogenic shock. Eur Heart J 2010; 31: 3032-3039.

7 Ponikowski P, Voors AA, Anker SD, et al. 2016 ESC Guidelines for the diagnosis and treatment of acute and chronic heart failure. Eur J Heart Fail 2016; 18: 891-975.

8 Sieweke JT, Berliner D, Tongers J, et al. Mortality in patients with cardiogenic shock treated with the Impella CP microaxial pump for isolated left ventricular failure. Eur Heart J Acute Cardiovasc Care 2020; 9: 138-148.

9 Schmidt M, Burrell A, Roberts L, et al. Predicting survival after ECMO for refractory cardiogenic shock: the survival after veno-arterial-ECMO (SAVE)-score. Eur Heart J 2015; 36: 2246-2256.

10 Reyentovich A, Barghash MH, Hochman JS. Management of refractory cardiogenic shock. Nat Rev Cardiol 2016; 13: 481-492.

11 Baran DA, Grines CL, Bailey S, et al. SCAI clinical expert consensus statement on the classification of cardiogenic shock. Catheter Cardiovasc Interv 2019; 94: 29-37.

12 Akin M, Sieweke JT, Zauner F, et al. Mortality in patients with out-of-hospital cardiac arrest undergoing a standardized protocol including therapeutic hypothermia and routine coronary angiography: experience from the HACORE registry. JACC Cardiovasc Interv 2018; 11: 1811-1820.

13 JCS Joint Working Group. Guidelines for diagnosis and treatment of myocarditis ( JCS 2009): digest version. Circ J 2011; 75: 734-743.

14 Napp LC, Kuhn C, Hoeper MM, et al. Cannulation strategies for percutaneous extracorporeal membrane oxygenation in adults. Clin Res Cardiol 2016; 105: 283-296.

15 Lin YH, Huang HC, Chang YC, et al. Multi-scale symbolic entropy analysis provides prognostic prediction in patients receiving extracorporeal life support. Crit Care 2014; 18: 548.

16 Tongers J, Sieweke JT, Kuhn C, et al. Early escalation of mechanical circulatory support stabilizes and potentially rescues patients in refractory cardiogenic shock. Circ Heart Fail 2020; 13: e005853.

17 Schafer A, Werner N, Westenfeld R, et al. Clinical scenarios for use of transvalvular microaxial pumps in acute heart failure and cardiogenic shock - A European experienced users working group opinion. Int J Cardiol 2019; 291: 96-104.

18 Werdan K, Gielen S, Ebelt H, et al. Mechanical circulatory support in cardiogenic shock. Eur Heart J 2014; 35: $156-167$.

19 Thiele H, Ohman EM, Desch S, et al. Management of cardiogenic shock. Eur Heart J 2015; 36: 1223-1230.

20 Pappalardo F, Schulte C, Pieri M, et al. Concomitant implantation of Impella on top of veno-arterial extracorporeal membrane oxygenation may improve survival of patients with cardiogenic shock. Eur J Heart Fail 2017; 19: 404-412.

21 Morris K, Patel A, Chaudhry SP, et al. An uncommon cause of fulminant myocarditis: influenza B. JACC 2019; 73: Suppl. 1, 2189.

22 Tschope C, Van Linthout S, Klein O, et al. Mechanical unloading by fulminant myocarditis: LV-IMPELLA, ECMELLA, BI-PELLA, and PROPELLA concepts. J Cardiovasc Transl Res 2019; 12: 116-123.

23 Matias G, Taylor R, Haguinet F, et al. Estimates of mortality attributable to influenza and RSV in the United States during 1997-2009 by influenza type or subtype, age, cause of death, and risk status. Influenza Other Respir Viruses 2014; 8: 507-515.

24 Paddock CD, Liu L, Denison AM, et al. Myocardial injury and bacterial pneumonia contribute to the pathogenesis of fatal influenza B virus infection. J Infect Dis 2012; 205: 895-905.

25 Esposito ML, Kapur NK. Acute mechanical circulatory support for cardiogenic shock: the "door to support" time. F1000Res 2017; 6: 737.

26 Pan HY, Yano M, Kido H. Effects of inhibitors of Toll-like receptors, protease-activated receptor-2 signalings and trypsin on influenza A virus replication and upregulation of cellular factors in cardiomyocytes. J Med Invest 2011; 58: 19-28.

27 Mamas MA, Fraser D, Neyses L. Cardiovascular manifestations associated with influenza virus infection. Int $J$ Cardiol 2008; 130: 304-309.

28 Mahrholdt H, Goedecke C, Wagner A, et al. Cardiovascular magnetic resonance assessment of human myocarditis: a comparison to histology and molecular pathology. Circulation 2004; 109: 1250-1258.

29 Yang X, Yu Y, Xu J, et al. Clinical course and outcomes of critically ill patients with SARS-CoV-2 pneumonia in Wuhan, China: a single-centered, retrospective, observational study. Lancet Respir Med 2020; 8: 475-481.

30 Chen C, Zhou Y, Wang DW. SARS-CoV-2: a potential novel etiology of fulminant myocarditis. Herz 2020; 45: $230-232$.

31 Zheng YY, Ma YT, Zhang JY, et al. COVID-19 and the cardiovascular system. Nat Rev Cardiol 2020; 17: 259-260.

32 Guo T, Fan Y, Chen M, et al. Cardiovascular implications of fatal outcomes of patients with coronavirus disease 2019 (COVID-19). JAMA Cardiol 2020; 5: 811-818.

33 Shi S, Qin M, Shen B, et al. Association of cardiac injury with mortality in hospitalized patients with COVID-19 in Wuhan. JAMA Cardiol 2020; 5: 802-810.

34 Inciardi RM, Lupi L, Zaccone G, et al. Cardiac involvement in a patient with coronavirus disease 2019 (COVID-19). JAMA Cardiol 2020; 5: 819-824.

35 Chen N, Zhou M, Dong X, et al. Epidemiological and clinical characteristics of 99 cases of 2019 novel coronavirus pneumonia in Wuhan, China: a descriptive study. Lancet 2020; 395: 507-513.

36 Modin D, Jorgensen ME, Gislason G, et al. Influenza vaccine in heart failure. Circulation 2019; 139 : 575-586.

37 Heinze G, Juni P. An overview of the objectives of and the approaches to propensity score analyses. Eur Heart $J$ 2011; 32: 1704-1708. 